\title{
Human Respiration Localization Method Using UWB Linear Antenna Array
}

\author{
Yuan Liu, ${ }^{1,2}$ Shiyou Wu, ${ }^{1}$ Jie Chen, ${ }^{1}$ Guangyou Fang, ${ }^{1}$ and Hejun Yin $^{3}$ \\ ${ }^{1}$ Key Laboratory of Electromagnetic Radiation and Sensing Technology, Institute of Electronics, Chinese Academy of Sciences, \\ Beijing 100190, China \\ ${ }^{2}$ University of Chinese Academy of Sciences, Beijing 100049, China \\ ${ }^{3}$ Chinese Academy of Sciences, Beijing 100864, China
}

Correspondence should be addressed to Jie Chen; chenjie@mail.ie.ac.cn

Received 17 October 2014; Revised 21 January 2015; Accepted 21 January 2015

Academic Editor: Chenzhong Li

Copyright (C) 2015 Yuan Liu et al. This is an open access article distributed under the Creative Commons Attribution License, which permits unrestricted use, distribution, and reproduction in any medium, provided the original work is properly cited.

Human respiration is the basic vital sign in remote monitoring. There has been remarkable progress in this area, but some challenges still remain to obtain the angle-of-arrival (AOA) and distinguish the individual signals. This paper presents a $2 \mathrm{D}$ noncontact human respiration localization method using Ultra-Wideband (UWB) 1D linear antenna array. The imaging reconstruction based on beamforming is used to estimate the AOA of the human chest. The distance-slow time 2D matrix at the estimated AOA is processed to obtain the distance and respiration frequency of the vital sign. The proposed method can be used to isolate signals from individual targets when more than one human object is located in the surveillance space. The feasibility of the proposed method is demonstrated via the simulation and experiment results.

\section{Introduction}

Impulse Ultra-Wideband (UWB) radar technology has become more and more attractive to the researchers of microwave sensing and imaging. It offers the advantages of high range resolution and good penetration for nonmetallic materials. It has been implemented in various systems for different applications, such as ground penetrating radar, through-wall imaging, and concealed weapon detection [1]. In recent years, it has become a promising technique for noncontact detection of human respiration which is the basic vital sign for health monitoring, disaster search-and-rescue, law enforcement, and urban warfare. A typical human respiration causes chest displacement from $0.1 \mathrm{~mm}$ to several millimeters depending on the target.

The conventional measurement system consists of two UWB antennas for transmission and reception, respectively [2]. When two or more targets are located in the field of view, it will be more challenging to isolate signals from individual targets. It generally extracts the respiration frequency and range without the information of the angle-of-arrival (AOA). Another challenge is the interference caused by the random body movement, which results in low signal-to-noise ratio (SNR) in practical applications.

Array-based systems have been used to improve the performance of vital sign estimation. Li and Lin [3] used 2 transceivers, one in front of and the other behind the human body, to cancel out $1 \mathrm{D}$ random body movement. Yu et al. [4] placed 4 transceivers at 4 sides of the human body to cancel out $2 \mathrm{D}$ random body movement and improve detection sensitivity. Akiyama et al. [5] used the system with one transmitting antenna and 4 receiving antennas to improve the SNR with correlation processing. Takeuchi et al. [6] utilized the value of correlation coefficient to measure the $3 \mathrm{D}$ location with $2 \mathrm{D}$ array antennas. Su et al. [7] determined AOA of human target from the intersection of AOA information with two phased array Doppler radars.

In this paper, a new method was proposed to determine AOA and separate useful scattered signals from multiple individuals with 1D linear antenna array. Modified Kirchhoff imaging method based on beamforming was used to combine the information from the multiple antennas at given slowtime frame. The AOA of human respiration was estimated from the reconstructed images of all slow-time frames. Then 
we evaluated the respiration frequency and distance at the estimated AOA. Even if individual respiration frequencies and distances were very similar, it would be possible to distinguish different human targets based on AOA. The performance of the proposed method was validated by simulation and experiment for SIMO system.

The rest of this paper is organized as follows. Section 2 describes the vital sign model for impulse UWB radar system. In Section 3, a description of the algorithm implementation and the data processing steps are given. Section 4 presents the numerical simulations that have been carried out for testing this algorithm. Moreover, experimental results are presented in Section 5. Finally, Section 6 concludes this paper.

\section{UWB Vital Sign Model}

The vital sign is detected by observing the change of roundtrip time of reflected signal from human chest. The round-trip delay of human chest in fast time is assumed to be a sinusoidal function:

$$
\tau_{r}(t)=\tau_{0}+\tau_{d} \sin \left(2 \pi f_{r} t\right),
$$

where $\tau_{0}$ is the round-trip delay of the chest vibration center, $\tau_{d}$ is the time corresponding to the displacement amplitude of respiration, $f_{r}$ is the respiration frequency, and $t$ indicates the slow time which corresponds to the acquisition time of distance profiles.

For impulse UWB radar, $u(\tau)$ is the transmitted impulse signal, and $\tau$ represents the fast time which corresponds to the distance dimension and is orthogonal to the slowtime dimension. The received signal $R(\tau, t)$ with respiration motion of one human target can be expressed as $[2,8]$

$$
\begin{aligned}
R(\tau, t)= & u\left(\tau-\tau_{r}(t)\right) * h_{r}(\tau) \\
& +\sum_{p=1, p \neq r}^{P} u\left(\tau-\tau_{p}\right) * h_{p}(\tau)+w(\tau),
\end{aligned}
$$

where $*$ denotes convolution, $h_{r}(\tau)$ is the impulse response of respiration motion, and $h_{p}(\tau)$ is the joint impulse response of transmitting antenna, receiving antenna, and $p$ th static object. $\tau_{p}$ is the round-trip delay of pth static object. $w(\tau)$ is the additive Gaussian white noise.

The captured signal is digitized and saved in a $2 \mathrm{D}$ data set denoted by $R[m, n]$ :

$$
R[m, n]=h[m, n]+c[m, n]+w[m, n],
$$

where $m=0,1, \ldots, M-1$ represents the fast-time index and $n=0,1, \ldots, N-1$ introduces the slow-time index. The signal is sampled at fast-time sampling interval of $\delta_{\tau}$. The slow time is discretized with $t=n T_{s}$, and $T_{s}$ is slow-time sampling interval. $h[m, n]$ denotes the response of respiration motion. $c[m, n]$ denotes the static clutter due to the reflected signals from static objects and the coupling between transmitting and receiving antennas. $w[m, n]$ denotes the additive Gaussian white noise.

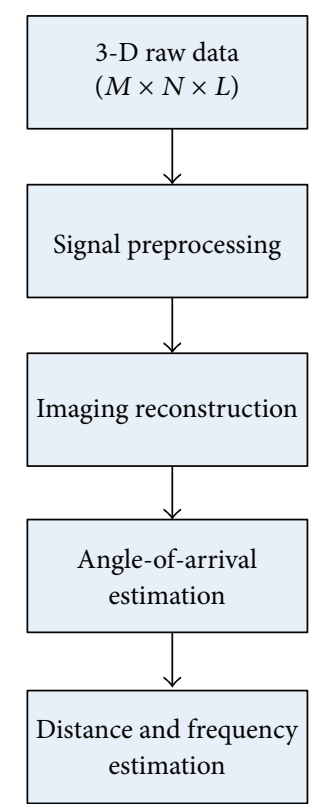

FIGURE 1: Flowchart of respiration detection algorithm.

For linear antenna array with $L_{T x}$ transmitting antennas and $L_{R x}$ receiving antennas, the number of the equivalent radar channels is equal to $L=L_{T x} \times L_{R x}$. The data set will be extended to a $3 \mathrm{D}$ matrix $R[m, n, l]$, where $l=0,1, \ldots, L-1$ indicates that the data is captured by the $l$ th radar channel.

\section{Detection Algorithm}

In this section, the algorithm is proposed to obtain vital information by UWB linear array. The implement steps of the algorithm are illustrated in the flowchart shown in Figure 1. The algorithm consists of four main steps.

3.1. Signal Preprocessing. The implement steps of signal preprocessing for each radar channel are illustrated in the flowchart shown in Figure 2. The preprocessing consists of three steps.

3.1.1. DC Offset Removal. Since the sampling gate of the impulse UWB receiver exhibits some DC offset, DC offset removal is necessary to ensure that the mean value in fasttime dimension is near to zero. Assume that the amplitude probability distribution in fast-time dimension is symmetric about the mean value and the short time mean value is constant over the time window. A simple way to remove DC offset is processed by subtracting the mean value over the fasttime window:

$$
R^{\prime}[m, n]=R[m, n]-\frac{1}{M} \sum_{m=0}^{M-1} R[m, n] .
$$

3.1.2. Linear Trend Suppression. The data acquisition typically accompanies time drift caused by the instability of the triggering unit in impulse UWB radar. It generally results in 


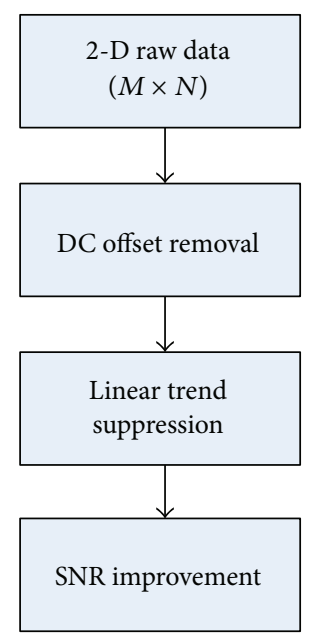

FIGURE 2: Flowchart of signal preprocessing.

linear trend in slow time. Linear trend suppression (LTS) method is used to remove the linear trend in $R[m, n]$ [9]. The potential linear trend in the slow-time dimension is estimated by means of a linear least-square fit. The result matrix $\widetilde{R}[m, n]$ is subsequently obtained by subtracting the estimated potential linear trend from $R^{\prime}[m, n]$ :

$$
\widetilde{\mathbf{R}}^{T}=\mathbf{R}^{\prime T}-\mathbf{x}\left(\mathbf{x}^{T} \mathbf{x}\right)^{-1} \mathbf{x}^{T} \mathbf{R}^{\prime T},
$$

where $\mathbf{x}=\left[n / N, 1_{N}\right], n=[0,1, \ldots, N-1]^{T}, 1_{N}$ is $N \times 1$ vector containing unit values.

3.1.3. SNR Improvement. A moving average is applied along the fast-time and slow-time dimensions for SNR improvement. It is commonly used to suppress the high-frequency clutter interferences. In addition, the high-frequency noise is often caused by the oversampling of backscattered signal. So an infinite impulse response (IIR) band-pass filtering on the data set $\widetilde{\mathbf{R}}$ in fast-time dimension is also performed as needed.

3.2. Imaging Reconstruction. Based on "time delay-and-sum" beamforming, the conventional back-projection (BP) algorithm has been successfully implemented in UWB imaging reconstruction [10]. In this method, the output is formed by summing delayed versions of the received signals. The delays are determined by the array geometry and the desired scanning angle. This approach is straightforward and easy to implement. However, it has some undesirable characteristics such as high side-lobes because it is not based on the rigorous wave theory.

An alternative to BP algorithm is Kirchhoff migration [11], which is based on wave equation. Conventional Kirchhoff migration is only feasible for monostatic array. It is not applicable to use conventional Kirchhoff migration for SIMO/MIMO UWB array imaging. Modified Kirchhoff migration was developed for MIMO UWB array in [12]. For
1-D SIMO linear array, the modified Kirchhoff migration is formulated in Cartesian coordinate as

$$
I_{t}(\mathbf{r})=\int\left(\cos \varphi_{1}+\cos \varphi_{2}\right) \frac{d_{1} d_{2}}{v} \frac{\partial}{\partial \tau} R\left(\frac{d_{1}+d_{2}}{v}, t, \mathbf{r}^{\prime}\right) d x^{\prime},
$$

where $\mathbf{I}_{t}$ is the reconstructed image at slow-time $t . d_{1}$ and $d_{2}$ denote the propagation distance from migrated point $\mathbf{r}(x, y)$ to the transmitting and receiving antennas, respectively. $\mathbf{r}^{\prime}\left(x^{\prime}, y^{\prime}\right)$ represents the location of receiving antenna. $R\left(\tau, t, \mathbf{r}^{\prime}\right)$ is the received signal at $\mathbf{r}^{\prime}$ for slow time $t . \varphi_{1}$ is the geometric angle between the distance direction and the transmitting antenna. $\varphi_{2}$ is the geometric angle between the distance direction and the receiving antenna. $v$ is the velocity of electromagnetic wave in the medium.

In this paper, the polar coordinate is used to estimate AOA of human chest. The pole is located at the center of the linear array. The surveillance space is divided into pixels by the distance and angle. At given slow-time frame $n, L$ radar channels are used to reconstruct the image of targets. The imaging intensity $I_{n}\left[\varphi_{p}, r_{q}\right]$ at migrated point $\mathbf{r}\left(\varphi_{p}, r_{q}\right)$ in polar coordinate can be calculated using the discrete formulation of (6):

$$
\begin{gathered}
I_{n}\left[\varphi_{p}, r_{q}\right]=\sum_{l=0}^{L-1}\left(\cos \varphi_{T x_{l}}+\cos \varphi_{R x_{l}}\right) \\
\cdot \frac{d_{T x_{l}}\left(\varphi_{p}, r_{q}\right) d_{R x_{l}}\left(\varphi_{p}, r_{q}\right)}{v} \Delta R[k, n, l], \\
k=\text { floor }\left(\frac{d_{T x_{l}}\left(\varphi_{p}, r_{q}\right)+d_{R x_{l}}\left(\varphi_{p}, r_{q}\right)}{v \delta_{\tau}}\right),
\end{gathered}
$$

where $\varphi_{p}(p=0,1, \ldots, P-1)$ denotes the polar angle and $r_{q}(q=0,1, \ldots, Q-1)$ denotes the radial distance. $l$ indicates the $l$ th radar channel. For the $l$ th radar channel, $\varphi_{T x_{I}}$ represents the geometric angle between the distance direction and the transmitting antenna. $\varphi_{R x_{l}}$ denotes the geometric angle between the distance direction and the receiving antenna. $d_{T x_{l}}\left(\varphi_{p}, r_{q}\right)$ is the propagation distance from the transmitting antenna to the imaging pixel. $d_{R x_{l}}\left(\varphi_{p}, r_{q}\right)$ is the propagation distance from the imaging pixel to the receiving antenna. The index $k$ locates the data at the correct time delay. floor $(x)$ means the largest integer not greater than $x . \Delta$ is finite difference along fast-time dimension. A complete image is reconstructed by calculating the image intensity of all the pixels.

The formation process of the slice of $3 \mathrm{D}$ matrix $S\left[r_{q}, n, \varphi_{p}\right]$ (distance, slow-time, and angle) at given angle $\varphi_{p}$ is shown in Figure 3. From the reconstructed image $I_{n}\left[\varphi_{p}, r_{q}\right]$ at given slow time $n$, we can get the distance profile at given angle $\varphi_{p}$ as shown in Figure 3(a). The slice of $\mathbf{S}$ at given angle $\varphi_{p}$ is created from the distance profiles for all slow-time indices. The vital sign of the human object might be in the interesting imaging cells of the radial zone, which is marked by the red dotted frame in Figure 3(a). The distance changes periodically due to the respiratory motion. This quasiperiodic variation along 


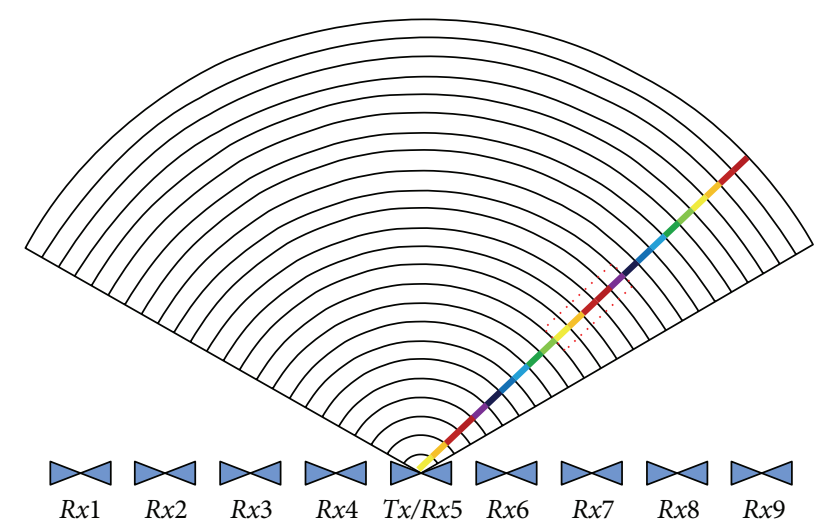

(a)

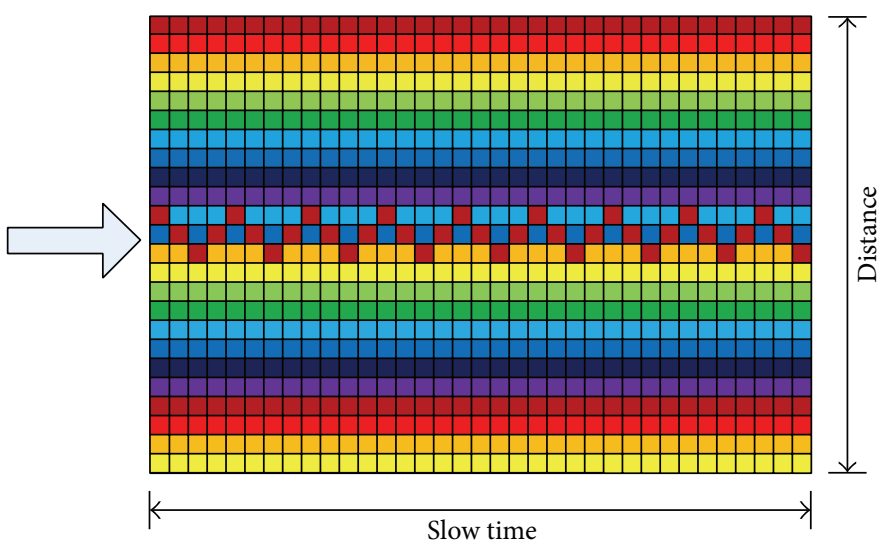

(b)

FIGURE 3: Formation of the slice of $\mathbf{S}$ at given angle.

slow time in the slice of $\mathbf{S}$ at given $\varphi_{p}$, as shown in Figure 3(b), can be used to estimate the respiratory frequency and distance.

\subsection{AOA Estimation}

3.3.1. Static Clutter Removal. Before AOA estimation, the static clutter needs to be removed from the slices of $\mathbf{S}$ for all angles. The signals reflected from human chest are very weak in comparison to the signals scattered from static objects and the coupling from the transmitting antenna to the receiving antennas. So it is essential to separate these weak signals from the static clutter.

There are different algorithms to remove the static clutter [13-15]. In the case of vital sign detection, the respiration response arrives mostly at the same round-trip delay in each measured signal and is misinterpreted as a static clutter by those algorithms. So the respiration response is almost undetectable with those algorithms. We suppress the static clutter using adaptive background subtraction method [16], which is based on the exponential averaging. The new estimate of static clutter $\mathbf{y}_{n}$ is calculated from the previous estimate $\mathbf{y}_{n-1}$ and the signal $\mathbf{x}_{n}$ :

$$
\mathbf{y}_{n}=\boldsymbol{\alpha}_{n} \mathbf{y}_{n-1}+\left(1-\boldsymbol{\alpha}_{n}\right) \mathbf{x}_{n},
$$

where $\boldsymbol{\alpha}_{n}$ is a vector of weighting coefficient with the size of $[Q \times 1] \cdot \mathbf{y}_{n}$ and $\mathbf{x}_{n}$ are $1 \mathrm{D}$ vectors with the size $[Q \times 1]$. $\mathbf{x}_{n}$ represents the distance profile in the matrix $S\left[r_{q}, n, \varphi_{p}\right]$ at given slow-time $n$ and angle $\varphi_{p}$. The vector of weighting coefficient $\boldsymbol{\alpha}_{n}$ is time-variant. Each element in $\boldsymbol{\alpha}_{n}$ is adaptively adjusted between 0 and 1 . Figure 4 shows an example of the result after removing the static clutter in Figure 3(b). As can be seen from Figure 3(b), the respiration response is blurred with the strong static clutter. In Figure 4, the static clutter is reduced and the respiration response is clearly highlighted as compared to Figure 3(b).

3.3.2. AOA Evaluation. After removing the static clutter, we can obtain the AOA of the human target from the peak value

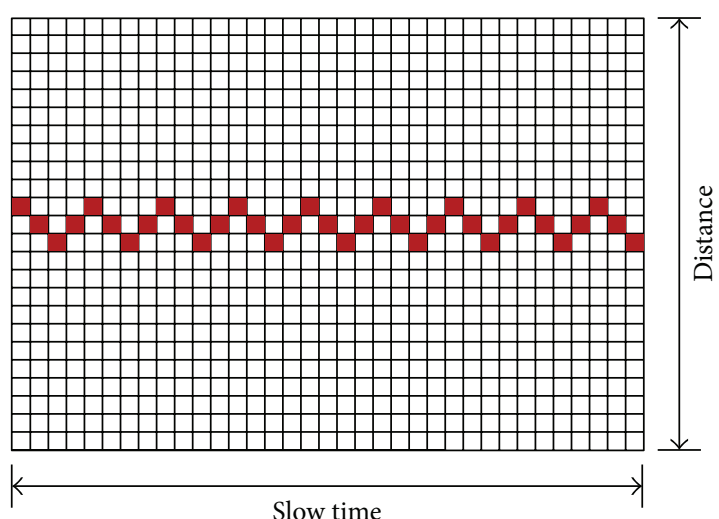

FIGURE 4: Result after removing the static clutter.

along angle dimension in the matrix $\mathbf{S}$. Assume that there is only one human object located in the surveillance space.

The matrix $E[n, p]$ with the size of $N \times P$ is obtained from the maximum value along the distance dimension in $S\left[r_{q}, n, \varphi_{p}\right]$ at given slow-time $n$ and angle $\varphi_{p}$. From $E[n, p]$, the AOA can be roughly evaluated from the index of the maximum value at given slow time $n$. In order to reduce the noise and avoid false evaluation, $E[n, p]$ is averaged along slow-time dimension:

$$
\tilde{E}[p]=\frac{1}{N} \sum_{n=0}^{N-1} E[p, n] .
$$

The AOA of the human target is given from the index of the maximum value in the vector $\widetilde{\mathbf{E}}$ :

$$
\theta=\theta_{0}+\underset{p}{\operatorname{argmax}}\{\widetilde{E}[p]\} \times \Delta \theta
$$

where $\Delta \theta$ is the interval of scanning angle and $\theta_{0}$ is the minimum scanning angle. 


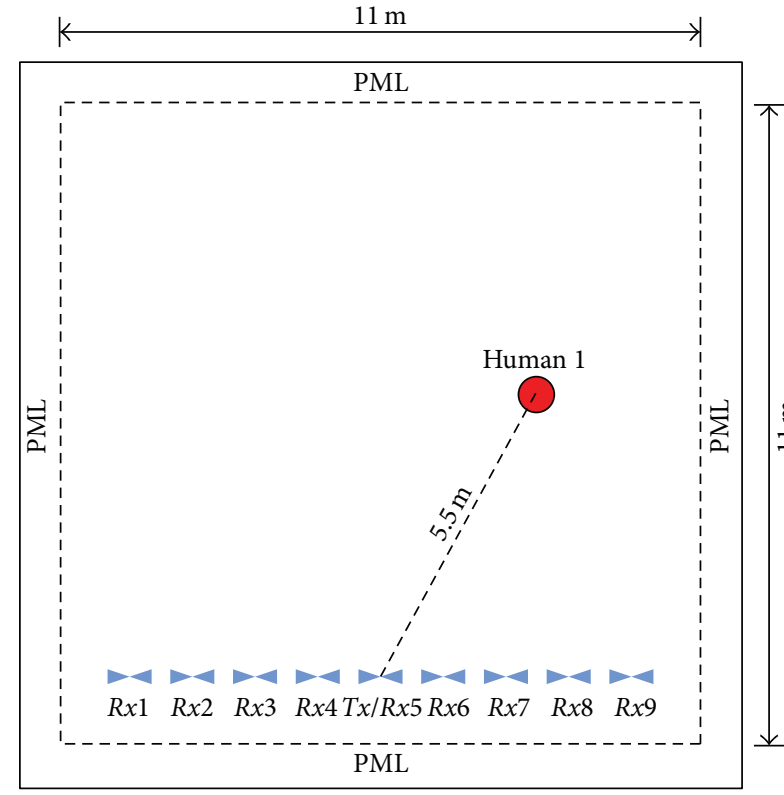

(a)

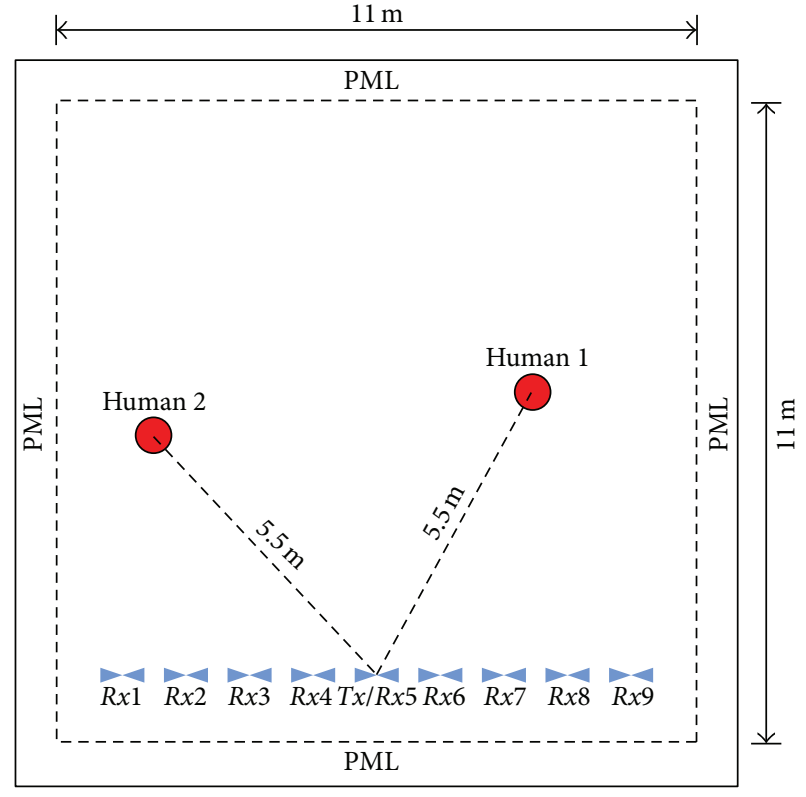

(b)

FIGURE 5: (a) FDTD model of first simulation. (b) FDTD model of second simulation.

When more than one human target is located in the surveillance space, the AOAs of human targets are estimated from the indices of the local maximum values of the vector $\widetilde{\mathbf{E}}$.

3.4. Distance and Frequency Estimation. The slice of the matrix $\mathbf{S}$ at the estimated AOA is processed to obtain the distance and respiration frequency using the method in [2]. Fourier transform is firstly performed in slow-time dimension, which generates the distance-frequency $2 \mathrm{D}$ matrix. The vital sign is identified in this matrix by using the method based on constant false alarm ratio (CFAR) and clustering.

\section{Simulations and Results}

4.1. Simulation Model and Parameters. With 2D finite-difference time-domain (FDTD) method, the received signals from the displacement of human chest were simulated for a SIMO system with one transmitting antenna and nine receiving antennas. The center antenna was used as both the transmitting and receiving antenna. The chest model was built as a metallic cylinder with the radius of $0.2 \mathrm{~m}$. The respiration motion of the chest was simulated as the periodic variation of the radius. The transmitted signal was the second derivative of Gaussian pulse with the center frequency of $400 \mathrm{MHz}$.

Two simulations were conducted to prove the performance of the proposed method. In the first simulation, the cylinder vibrated with the frequency of $0.5 \mathrm{~Hz}$. It was located at $5.5 \mathrm{~m}$ away from the center of antenna array at the angle of $60^{\circ}$ in free space. Another cylinder with the same vibrating frequency was added in the second simulation. It was located at the same distance but at a different angle of
TABLE 1: Simulation parameters.

\begin{tabular}{lcc}
\hline Parameters & Quantity & Value \\
\hline$D_{\text {width }}$ & The width of the simulated space & $11 \mathrm{~m}$ \\
$D_{\text {height }}$ & The height of the simulated space & $11 \mathrm{~m}$ \\
$R$ & Distance of chest center & $5.5 \mathrm{~m}$ \\
$f_{r}$ & Respiration frequency & $0.5 \mathrm{~Hz}$ \\
$f_{0}$ & Center frequency & $400 \mathrm{MHz}$ \\
$M$ & Fast-time sampling number & 2048 \\
$N$ & Slow-time sampling number & 512 \\
$\delta_{\tau}$ & Fast-time sampling interval & $40 \mathrm{ps}$ \\
\hline
\end{tabular}

$135^{\circ}$. Figure 5 shows the 2D FDTD model of two simulations. The simulation parameters are detailed in Table 1.

4.2. Simulation Results. Figure 6 shows the distance-frequency matrices for 9 channels using the respiration detection method [2] in the first simulation. The distances and respiration frequencies are extracted and shown in Figure 7. The respiration frequencies are the same for all channels (see Figure 7(b)), while the distances are different (see Figure $7(\mathrm{a})$ ).

The simulated data in first simulation is processed using the proposed method. Figure 8(a) plots the normalized values of each row in $E[n, p]$ along the scanning-angle dimension. From Figure 8(a), the AOA of the respiration is evaluated as $60^{\circ}$ by (10). The distance-slow time $2 \mathrm{D}$ matrix at the angle of $60^{\circ}$ is processed to estimate the distance and frequency. The distance-frequency matrix is shown in Figure 8(b). It shows that the distance is $5.5 \mathrm{~m}$ and the respiration frequency is $0.5 \mathrm{~Hz}$. 


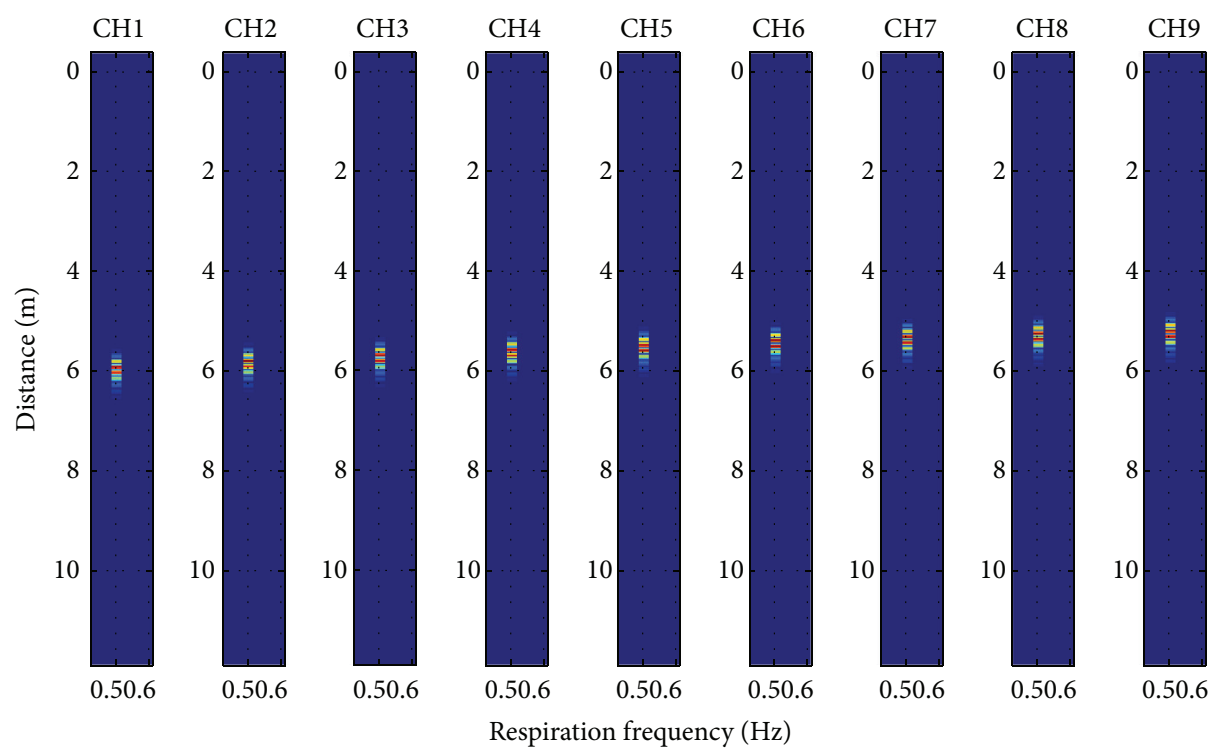

FIGURE 6: Distance-frequency matrices for all channels in the first simulation.

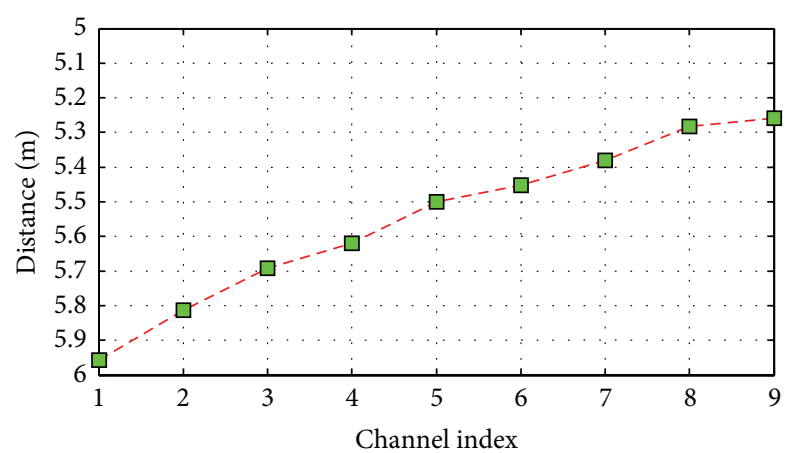

(a)

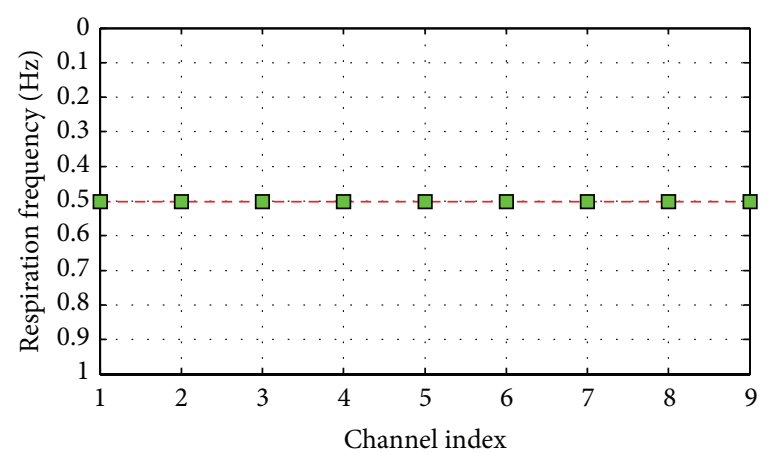

(b)

Figure 7: (a) The estimated distances for all channels. (b) The estimated respiration frequencies for all channels.

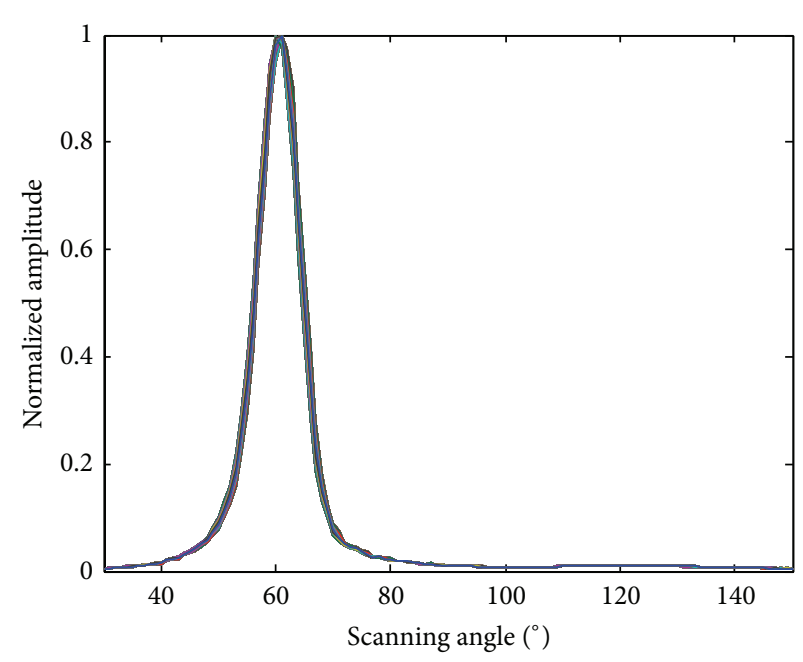

(a)

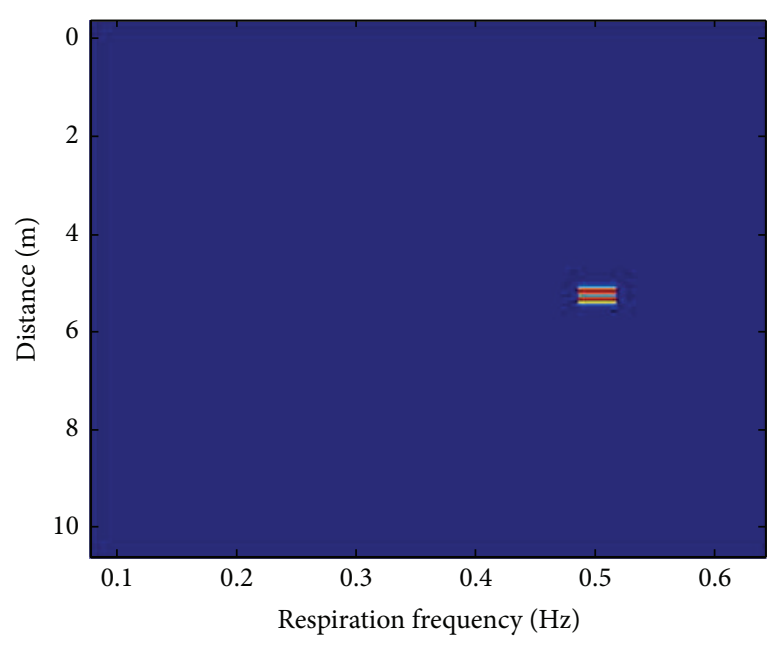

(b)

Figure 8: (a) The functions of the scanning angle in the first simulation. (b) The distance-frequency matrix at $60^{\circ}$. 


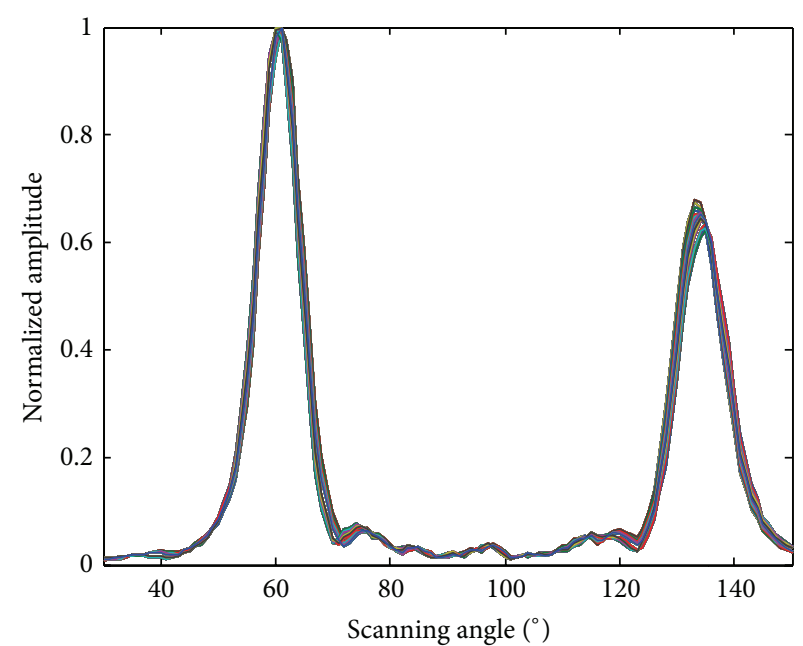

(a)

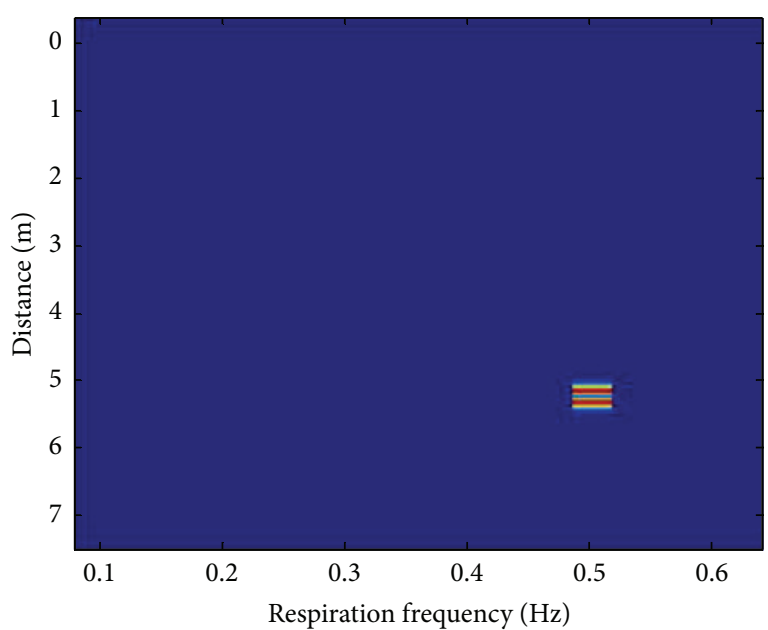

(b)

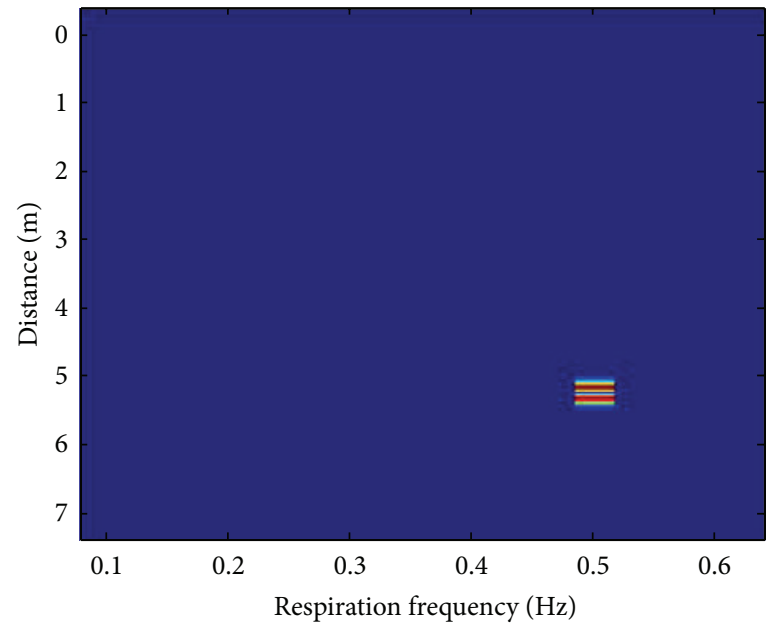

(c)

FIgURE 9: (a) The functions of the scanning angle in the second simulation. (b) The distance-frequency matrix at $60^{\circ}$. (c) The distance-frequency matrix at $135^{\circ}$.

For the second simulation, the functions of the scanning angle are plotted in Figure 9(a). Two peaks indicate that two targets are located at different angles. The corresponding AOAs are extracted as $60^{\circ}$ and $135^{\circ}$. The distance-frequency matrices for $60^{\circ}$ and $135^{\circ}$ are shown in Figures $9(\mathrm{~b})$ and $9(\mathrm{c})$. The distances and frequencies of two targets are estimated at $5.5 \mathrm{~m}$ and $0.5 \mathrm{~Hz}$. Even though the individual frequencies and distances are the same, the two targets can be distinguished by the AOAs with SIMO system.

\section{Experiment and Results}

5.1. Experimental Scenario. The measurement setup as shown in Figure 10 consists of Geozondas 8-channel digital sampling converter SD-10820, Geozondas pulse generator GZ1118GN03, linear antenna array, and PC. SD-10820 is used to trigger the pulse generator and sample the signal using sequential sampling technique. The channel bandwidth of SD-10820 is $0.2-20 \mathrm{GHz}$. A Gaussian pulse with $300 \mathrm{ps}$ duration and $50 \mathrm{~V}$ amplitude is sent by the pulse generator to the transmitting antenna. PC sets the parameters of the sampling converter and gets the digitized data via USB interface. The linear antenna array made up of 9 cavity-backed bowtie dipoles is used with an interelement space of $195 \mathrm{~mm}$. The element at the center of the linear array is used for the transmitting antenna. The other 8 elements are receiving antennas.

The radar parameters are shown in Table 2. During data acquisition, SNR was improved by averaging every 20 received signals. The total sampling points $M$ in fast-time dimension were set to 1000 . It took $48 \mathrm{~s}$ to acquire $N=512$ echoes for the measurement.

The scenario of the measurement is shown in Figure 11. Table 3 shows the locations of three human targets. 


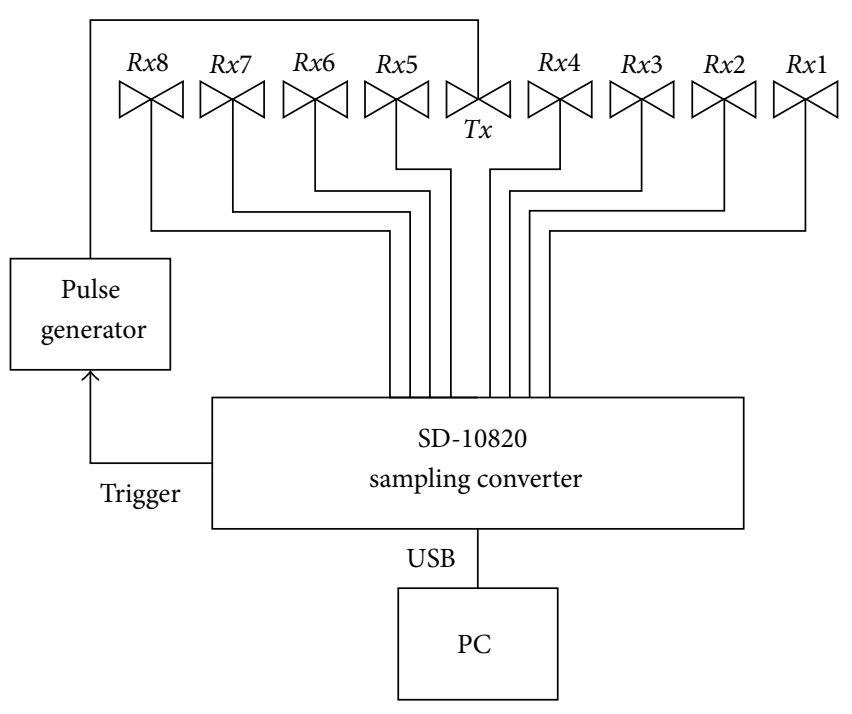

FIGURE 10: Experimental measurement setup.

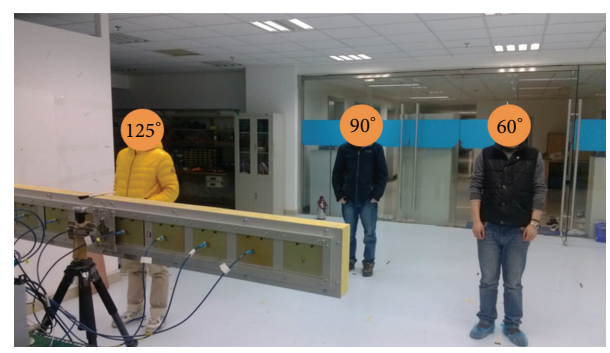

FIGURE 11: Experimental scenario for respiration detection of three human targets.

TABle 2: Parameters of the UWB radar system.

\begin{tabular}{lc}
\hline Parameters & Value \\
\hline Operating mode & Impulse \\
Antenna bandwidth & $0.5-2.5 \mathrm{GHz}$ \\
Antenna gain at $1.5 \mathrm{GHz}$ & $8.5 \mathrm{dBi}$ \\
Pulse repetition frequency & $500 \mathrm{KHz}$ \\
Time window & $40 \mathrm{~ns}$ \\
Sampling points $(M)$ & 1000 \\
ADC resolution & $14 \mathrm{bit}$ \\
\hline
\end{tabular}

TABLE 3: Target locations.

\begin{tabular}{lcc}
\hline & Distance & Angle \\
\hline Target 1 & $2.5 \mathrm{~m}$ & $60^{\circ}$ \\
Target 2 & $3.3 \mathrm{~m}$ & $90^{\circ}$ \\
Target 3 & $1.6 \mathrm{~m}$ & $125^{\circ}$ \\
\hline
\end{tabular}

5.2. Experimental Results. Figure 12 shows the distance-frequency matrices for each one of 8 channels using the respiration detection method in [2]. As can be seen in Figure 12, the vital sign with $0.42 \mathrm{~Hz}$ respiration frequency is clearly highlighted, which corresponds to target 2 . The respiration frequencies from target 1 and target 3 are blurred due to low SNR. From the amplitude and the distance of respiration responses, we can tell that target 1 is closer to the 1st channel, target 2 is located right ahead of the antenna array, and target 3 is closer to the 8th channel. But the exact AOAs cannot be evaluated from these matrices. The beamforming is used to coherently combine the signals from all 8 channels to extract the AOAs.

The functions of the scanning angle are plotted in Figure 13(a). Three groups of peak values obviously appear at the scanning angles $60^{\circ}, 90^{\circ}$, and $125^{\circ}$, respectively. Figure 13(b) gives the averaged and normalized function of the scanning angle, from which three estimated AOAs are $60^{\circ}$, $90^{\circ}$, and $125^{\circ}$.

The distance-slow time $2 \mathrm{D}$ matrices at the angles of $60^{\circ}$, $90^{\circ}$, and $125^{\circ}$ are obtained and used for the estimation of the distances and respiration frequencies. The distance-frequency matrix at the angle of $60^{\circ}$ is shown in Figure 14(a). The distance and respiration frequency can be estimated at $2.5 \mathrm{~m}$ and $0.2 \mathrm{~Hz}$. Similarly, the distance and respiration frequency can be estimated at $3.3 \mathrm{~m}$ and $0.42 \mathrm{~Hz}$ from the distance-frequency matrix at the angle of $90^{\circ}$ shown in Figure 14(b). For the distance-frequency matrix at the angle of $125^{\circ}$ shown in Figure 14(c), two vital signs are shown with one false sign. The false sign is identified and removed using the method in [2]. The distance and respiration frequencies are estimated at $1.6 \mathrm{~m}$ and $0.29 \mathrm{~Hz}$. Comparing Figure 14 with Figure 12, SNR of the distance-frequency matrix is improved, because the beamforming coherently combines the multiplechannel signals and obtains processing gain.

\section{Conclusion}

A novel respiration detection method based on UWB linear antenna array is presented in this paper. This method can be used to obtain the vital information, including the angleof-arrival and distance and respiration frequency. When more than one target is in the field of view, the proposed method can estimate the AOAs of individuals. Then we can distinguish the different sources of respiration motion, isolate the desired signals, and determine the distance and respiration frequency of individuals.

It is noted that the AOA estimation can perform well only on the assumption that no more than one human object is located at a specific scanning angle. Further efforts should be made to overcome this limitation and detect vital signs in complex environment.

\section{Conflict of Interests}

The authors declare that there is no conflict of interests regarding the publication of this paper. 


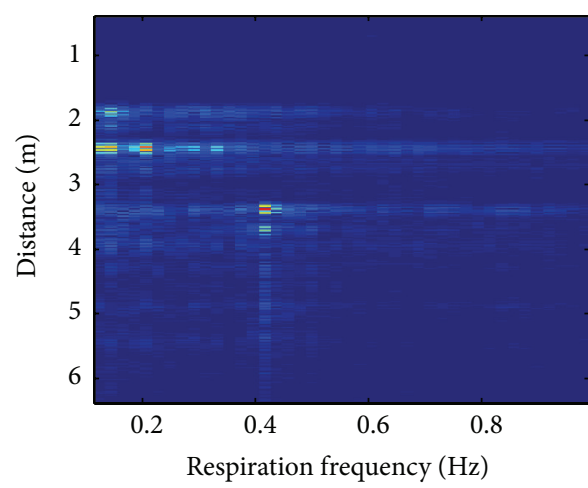

(a)

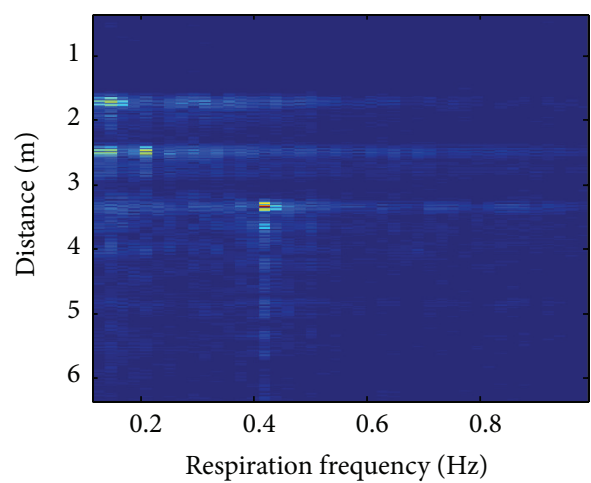

(c)

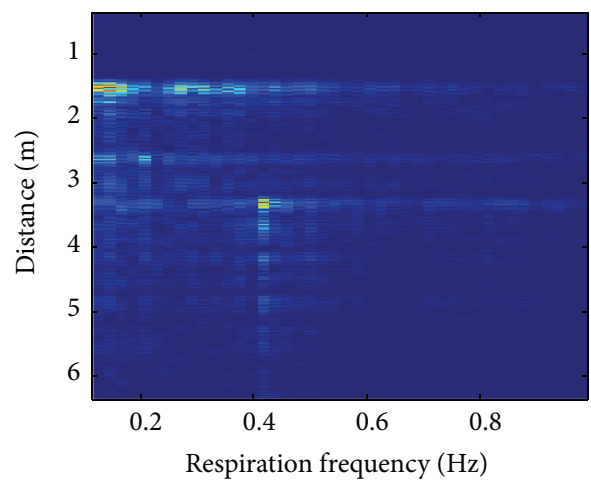

(e)

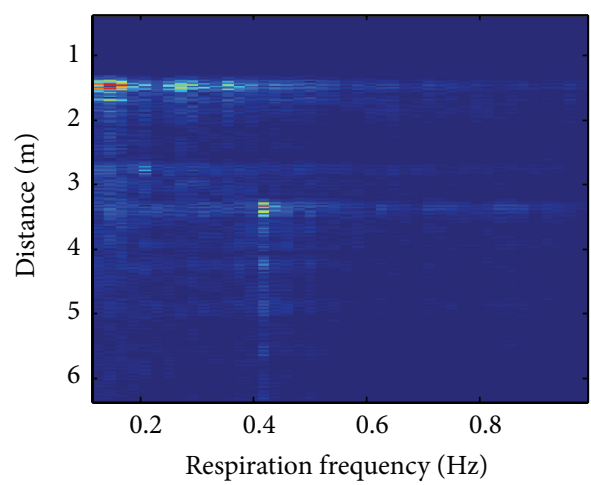

(g)

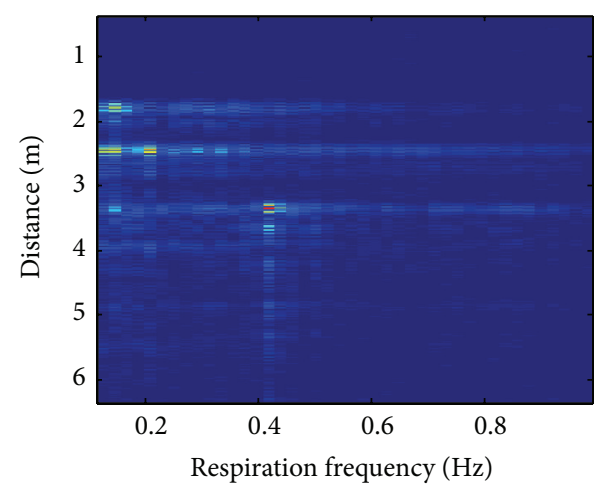

(b)

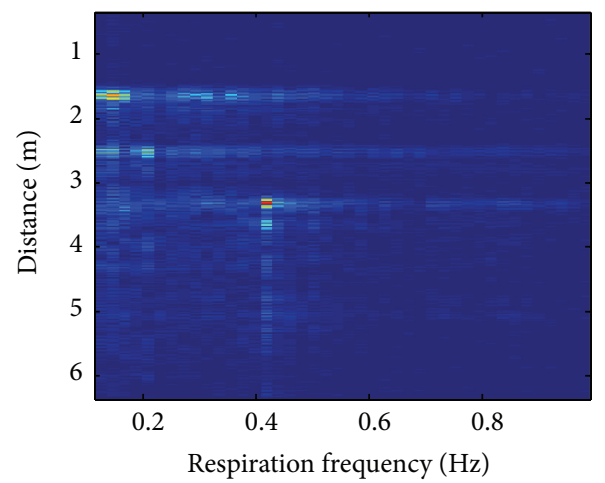

(d)

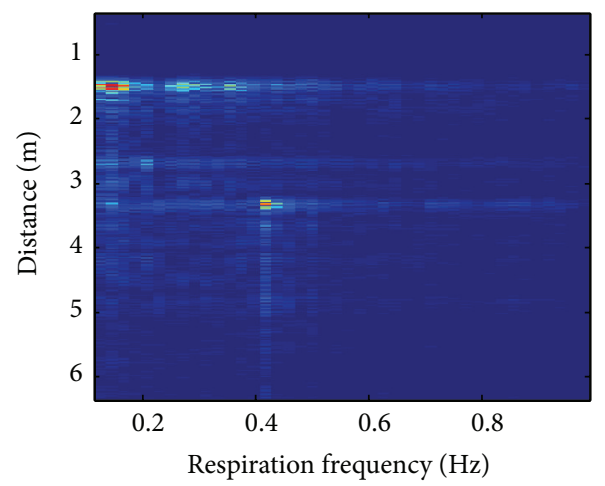

(f)

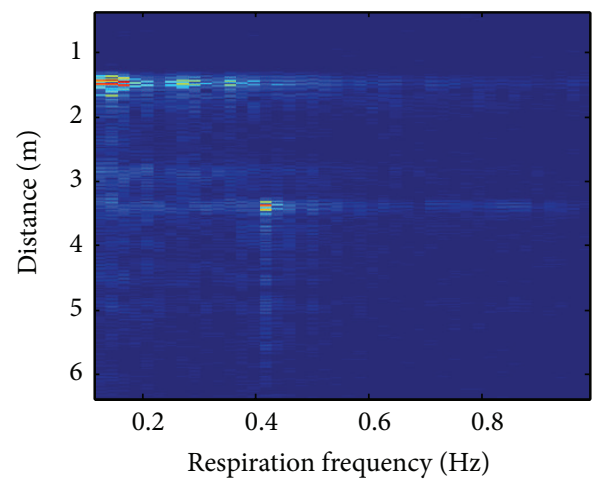

(h)

Figure 12: The range-frequency matrices for 8 channels. (a) The 1st channel. (b) The 2nd channel. (c) The 3rd channel. (d) The 4th channel. (e) The 5th channel. (f) The 6th channel. (g) The 7th channel. (h) The 8th channel. 


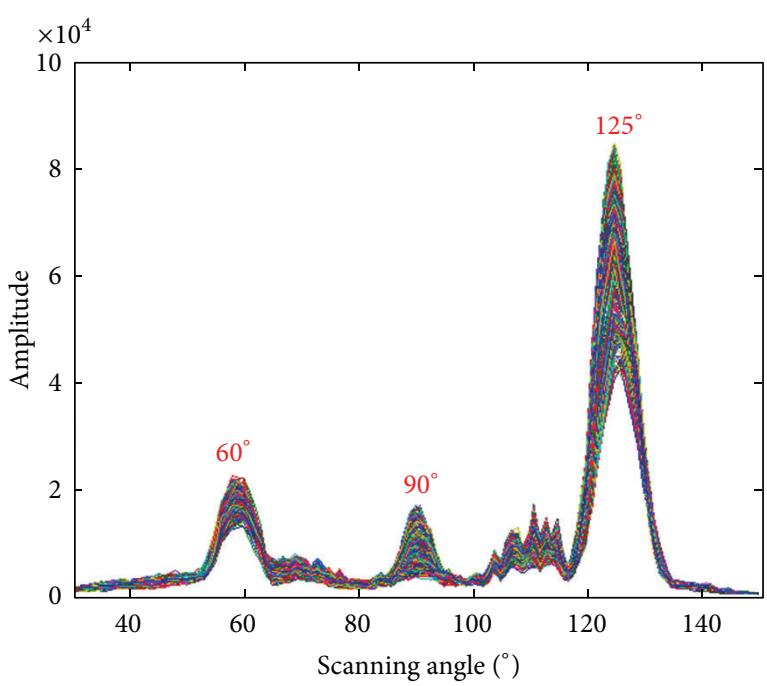

(a)

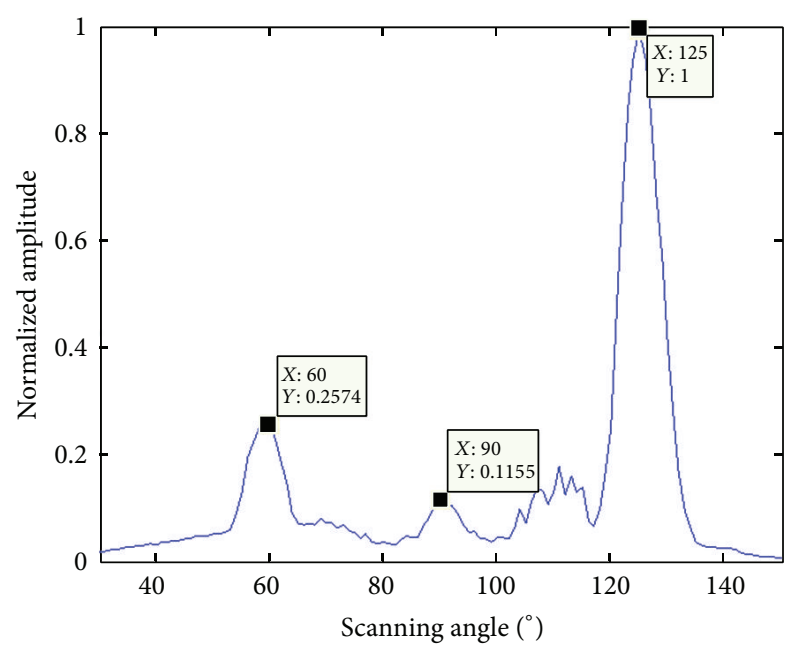

(b)

FIgURE 13: (a) The functions of the scanning angle. (b) The averaging and normalized function of the scanning angle.

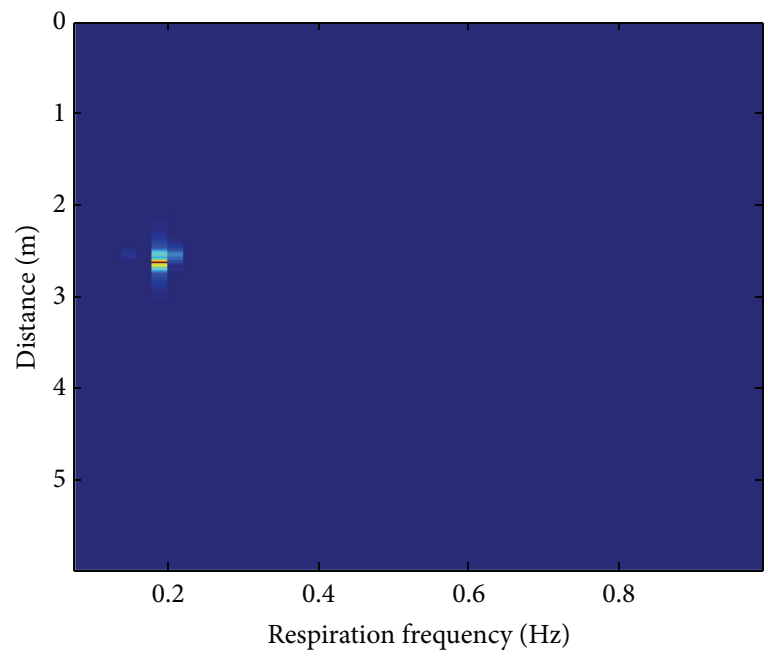

(a)

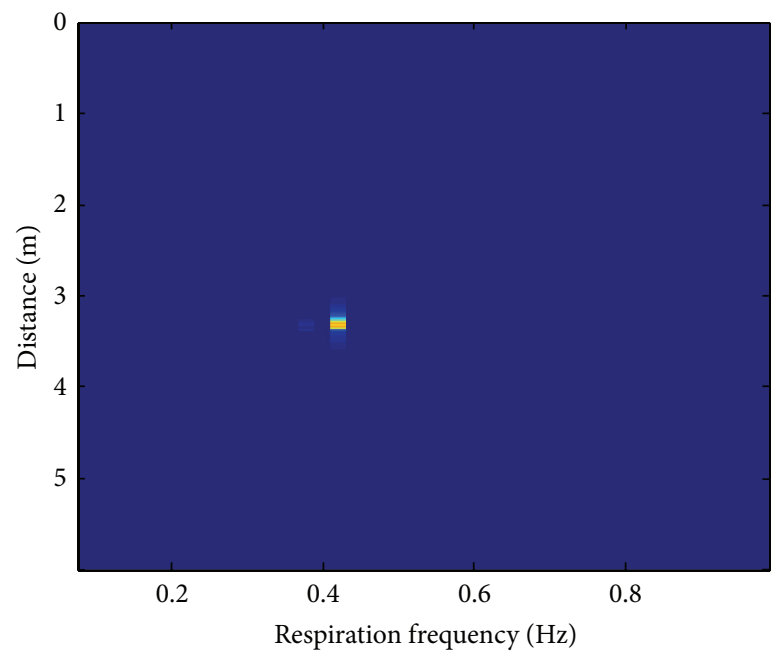

(b)

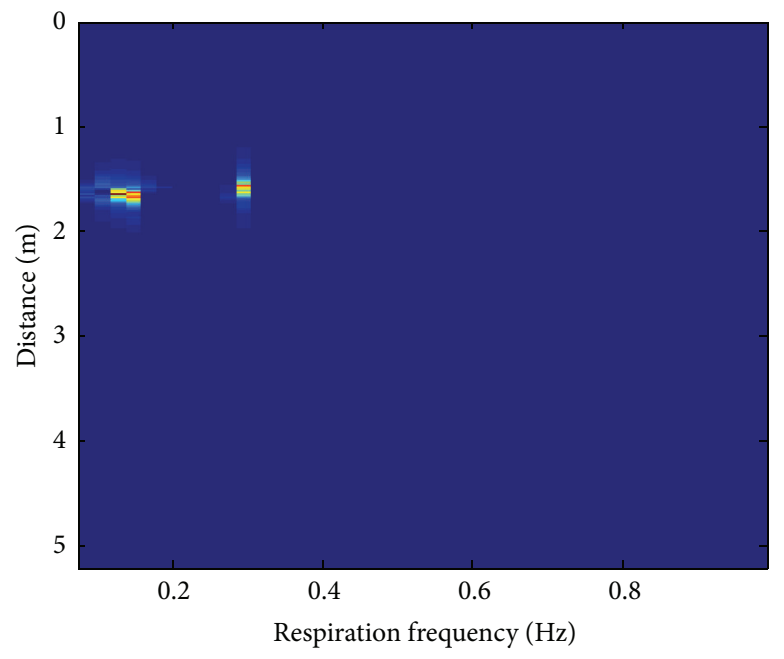

(c)

Figure 14: The distance-frequency matrices. (a) At the angle of $60^{\circ}$. (b) At the angle of $90^{\circ}$. (c) At the angle of $125^{\circ}$. 


\section{Acknowledgment}

Funding for this work was provided by the National High Technology Research and Development Program of China (863 Program) under Grant no. 2012AA061403.

\section{References}

[1] D. J. Daniels, EM Detection of Concealed Targets, John Wiley \& Sons, Hoboken, NJ, USA, 2009.

[2] Y. Xu, S. Wu, C. Chen, J. Chen, and G. Fang, "A novel method for automatic detection of trapped victims by ultrawideband radar," IEEE Transactions on Geoscience and Remote Sensing, vol. 50, no. 8, pp. 3132-3142, 2012.

[3] C. Li and J. Lin, "Complex signal demodulation and random body movement cancellation techniques for non-contact vital sign detection," in Proceedings of the IEEE MTT-S International Microwave Symposium Digest (MTT '08), pp. 567-570, IEEE, Atlanta, Ga, USA, June 2008.

[4] X. Yu, C. Li, and J. Lin, "Two-dimensional noncontact vital sign detection using Doppler radar array approach," in Proceedings of the IEEE MTT-S International Microwave Symposium (IMS '11), Baltimore, Md, USA, June 2011.

[5] I. Akiyama, N. Yoshizumi, A. Ohya, Y. Aoki, and F. Matsuno, "Search for survivors buried in rubble by rescue radar with array antennas-extraction of respiratory fluctuation," in Proceedings of the IEEE International Workshop on Safety, Security and Rescue Robotics (SSRR '07), pp. 1-6, Rome, Italy, September 2007.

[6] T. Takeuchi, H. Saito, Y. Aoki, A. Ohya, F. Matsuno, and I. Akiyama, "Rescue radar system with array antennas," in Proceedings of the 34th Annual Conference of the IEEE Industrial Electronics Society (IECON '08), pp. 1782-1787, Orlando, Fla, USA, November 2008.

[7] Y.-S. Su, C.-C. Chang, J.-J. Guo, and S.-F. Chang, "2-D wireless human subjects positioning system based on respiration detections," in Proceedings of the IEEE MTT-S International Microwave Symposium (IMS '12), pp. 1-3, IEEE, Montreal, Canada, June 2012.

[8] S. Venkatesh, C. R. Anderson, N. V. Rivera, and R. M. Buehrer, "Implementation and analysis of respiration-rate estimation using impulse-based UWB," in Proceedings of the IEEE Military Communications Conference (MILCOM '05), pp. 3314-3320, Atlantic City, NJ, USA, October 2005.

[9] A. Nezirović, A. G. Yarovoy, and L. P. Ligthart, "Signal processing for improved detection of trapped victims using UWB radar," IEEE Transactions on Geoscience and Remote Sensing, vol. 48, no. 4, pp. 2005-2014, 2010.

[10] M. D. Desai and W. K. Jenkins, "Convolution backprojection image reconstruction for spotlight mode synthetic aperture radar," IEEE Transactions on Image Processing, vol. 1, no. 4, pp. 505-517, 1992.

[11] N. Bleistein, "On the imaging of reflectors in the earth," Geophysics, vol. 52, no. 7, pp. 931-942, 1987.

[12] X. Zhuge, T. G. Savelyev, A. G. Yarovoy, and L. P. Ligthart, "UWB array-based radar imaging using modified kirehhoff migration," in Proceedings of the IEEE International Conference on UltraWideband (ICUWB '08), pp. 175-178, Hannover, Germany, September 2008.

[13] M. Piccardi, "Background subtraction techniques: a review," in Proceedings of the IEEE International Conference on Systems,
Man and Cybernetics (SMC '04), pp. 3099-3104, Hague, The Netherlands, October 2004.

[14] A. Mcivor, V. Zang, and R. Klette, "The background subtraction problem for video surveillance systems," in Proceedings of International Workshop on Robot Vision (RobVis '01), pp. 176183, Auckland, New Zealand, February 2001.

[15] A. G. Yarovoy, P. van Genderen, and L. P. Ligthart, "Ground penetrating impulse radar for landmine detection," in Proceedings of the 8th International Conference on Ground Penetrating Radar, pp. 856-860, Gold Coast, Australia, May 2000.

[16] R. Zetik, S. Crabbe, J. Krajnak, P. Peyerl, J. Sachs, and R. Thomä, "Detection and localization of persons behind obstacles using M-sequence through-the-wall radar," in Proceedings of the Sensors, and Command, Control, Communications, and Intelligence (C3I) Technologies for Homeland Security and Homeland Defense V, Orlando, Fla, USA, April 2006. 

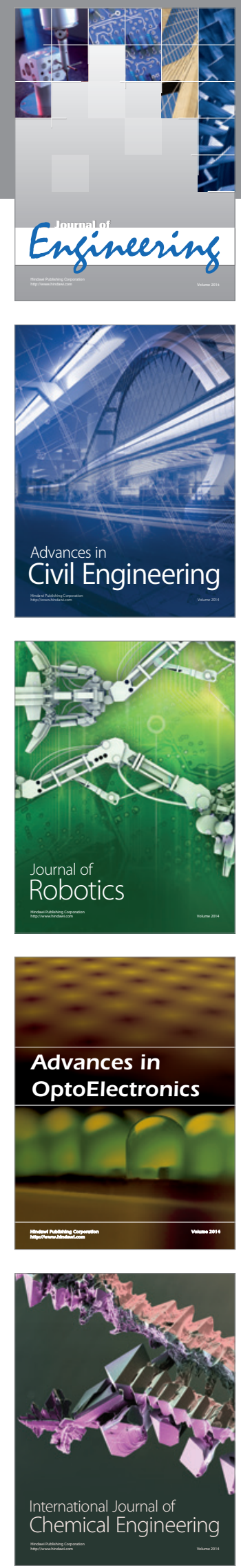

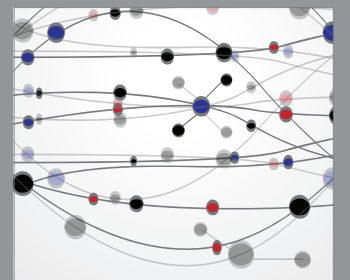

The Scientific World Journal
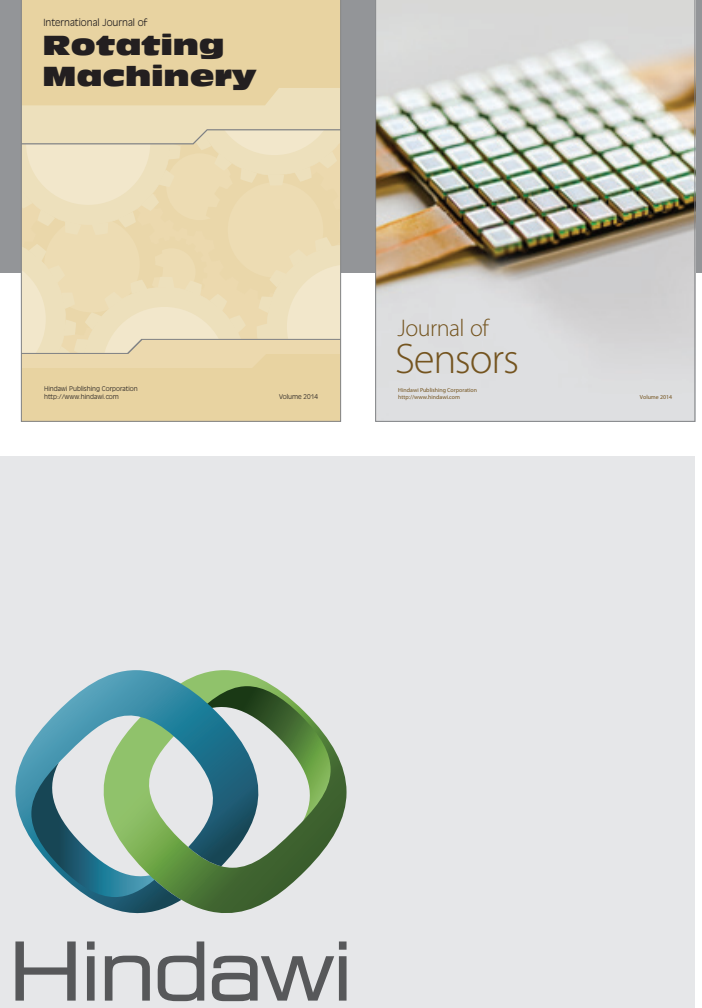

Submit your manuscripts at http://www.hindawi.com
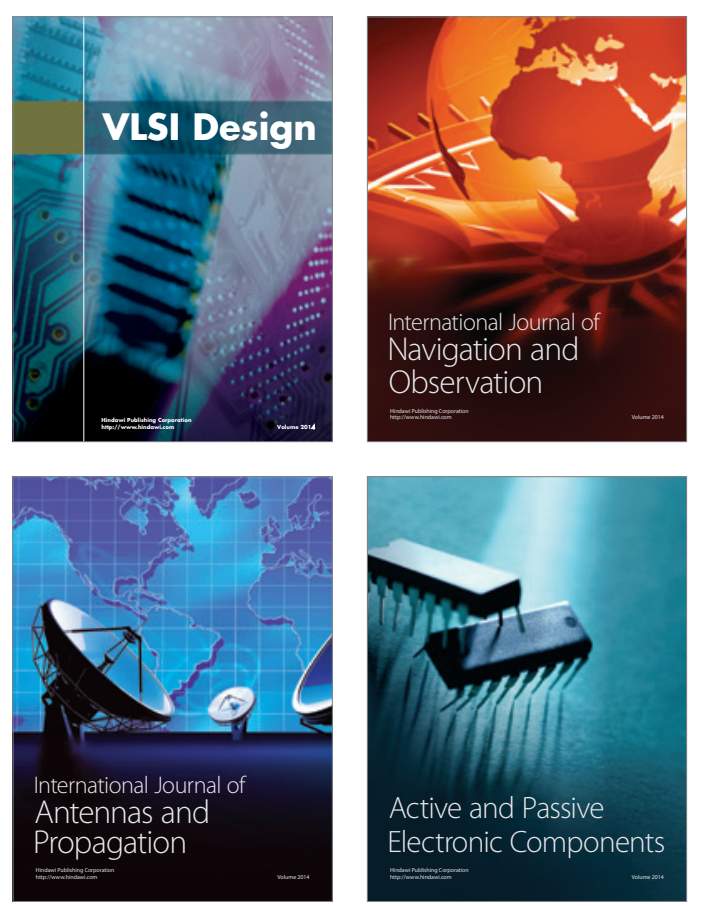
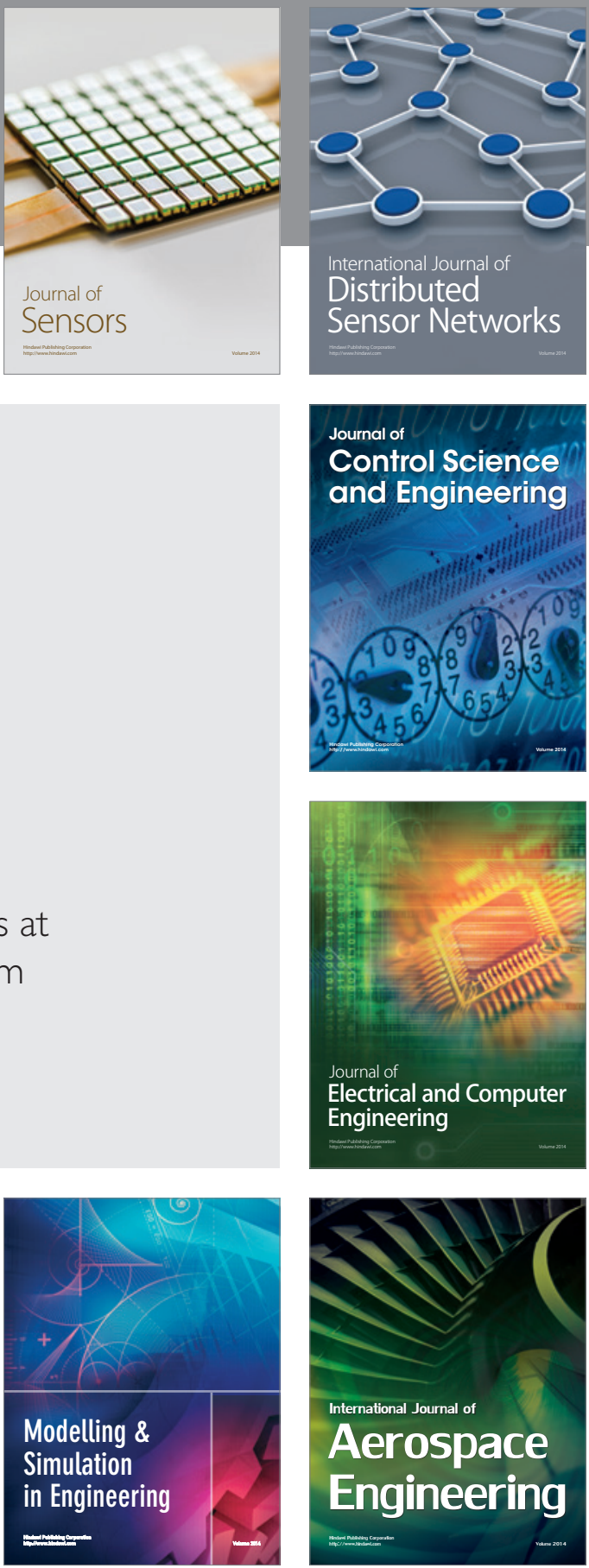

Journal of

Control Science

and Engineering
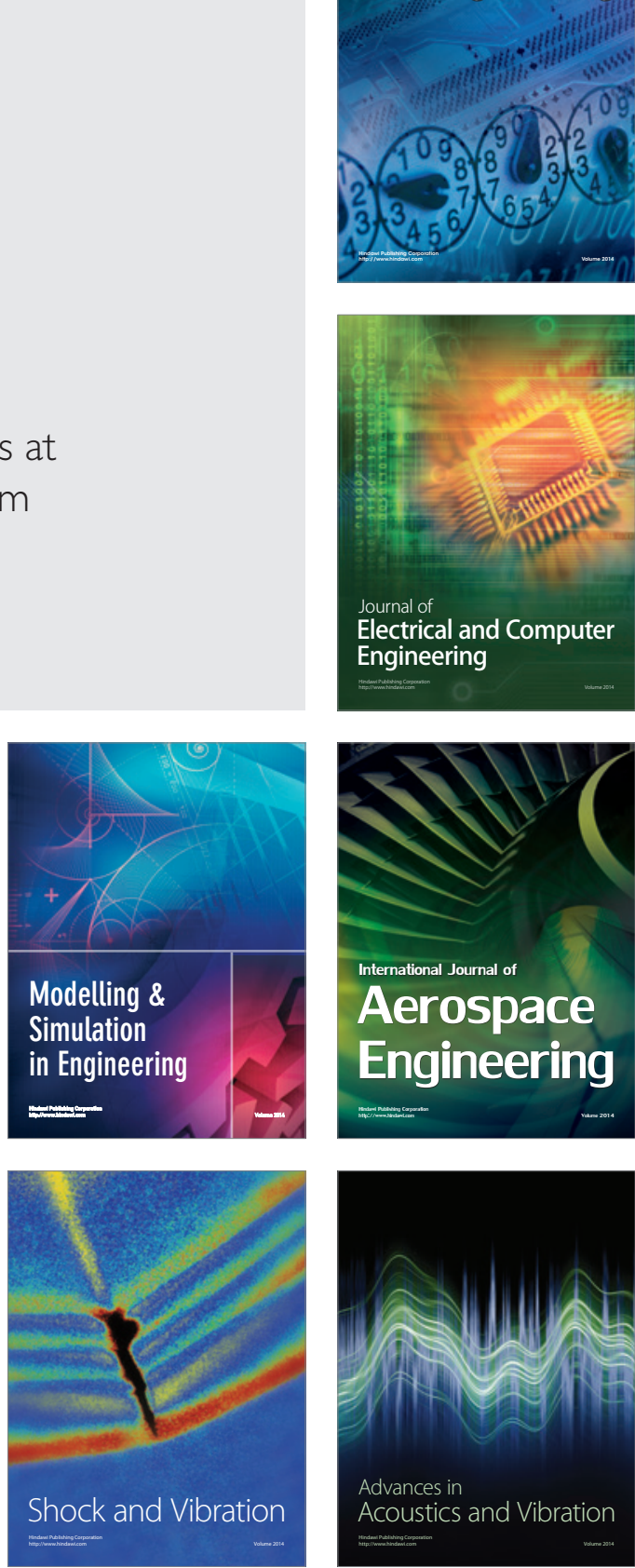